(SI)

\title{
Silylethynyl Substitution for Preventing Aggregate Formation in Perylene Diimides
}

\author{
Erkan Aksoy abc*, Andrew Danos ${ }^{\mathrm{b}}$, Chunyong Li ${ }^{\mathrm{b}}$, Andrew P. Monkman ${ }^{\mathrm{b} * *}$ and Canan \\ Varliklic \\ * erkanaksoy@iyte.edu.tr \\ **a.p.monkman@durham.ac.uk \\ a Solar Energy Institute, Ege University, 35100, Izmir, Turkey \\ bDepartment of Physics, Durham University, Durham DH1 3LE, U.K. \\ 'Department of Photonics, Izmir Institute of Technology, 35430, Urla, Izmir, Turkey
}




\section{General information:}

Hydrochloric acid (\%37), sodium hydroxide, trimethylsilyacetylene, triisopropylsilyacetylene, triphenylsilyacetylene, tetrakis(triphenylphosphine)palladium(0), bromine, hexane, chloroform, dichlorometane (DCM), N,N-dimethyl-formamide (DMF), silica gel 0.040$0.063 \mathrm{~mm}$, tetrabutylammonium hexafluorophosphate (TBAPF6) (\%99) were obtained from Sigma-Aldrich. Diisopropylamine, silica gel 60 F254 (thin-layer chromatography) and 2ethyl-1-hexylamine were obtained from Merck and perylene-3,4,9,10-tetracarboxylic dianhydride (PTCDA), copper(I) iodide were purchased from Fluka.

\section{Synthesis of perylene derivatives}

PDIref, PDI1, PDI2 and PDI3 were synthesized according to the literature. ${ }^{1-7}$

\section{Spectroscopic techniques}

\section{Steady state absorption and PL spectroscopy:}

In this study, an Analytik Jena S $600 \mathrm{UV}-\mathrm{Vis}$ spectrophotometer was used for absorption measurements. In addition, PL, optical stability test $\left(\mathrm{t}_{1 / 2}\right)$ and fluorescence quantum yield $(\Phi)$ measurements were performed in Edinburgh Instruments FLS920P spectrophotometer using an integrated sphere.

\section{Electrochemical properties:}

In cyclic voltammetry studies, glassy carbon, platinum wire, $\mathrm{Ag} / \mathrm{AgCl}$ and ferrocene (Fc) / ferrocenium were used as working electrode, counter electrode, reference electrode, and inner electrode, respectively. The scan rate was $100 \mathrm{mVs}^{-1}$. Dichloromethane solutions of $0.1 \mathrm{M}$ TBAPF6 were used as the support electrolyte. Before CV measurement of the PDIs, CV measurements of the solution medium containing only the support electrode were taken and the cleanliness of the medium was checked and the working range was determined. DCM solution of $0.1 \mathrm{M}$ TBAPF6 was found to be suitable for the PDI derivatives. ${ }^{8}$

\section{Time-resolved emission spectroscopy:}

Time-resolved spectra were recorded using nanosecond gated luminescence and lifetime measurements using a pulsed Nd:YAG laser emitting at $355 \mathrm{~nm}$ (EKSPLA) and pulse width $\sim 150$ ps. Emission from the samples was focused onto a spectrograph equipped with 300 lines/mm grating and $500 \mathrm{~nm}$ blaze wavelength and detected on a sensitive gated iCCD camera (Stanford Computer Optics 4Picos) with sub-nanosecond resolution. Time-resolved measurements were performed with variable CCD gate and delay times relative to the laser trigger, allowing emission decays (e.g. Figures S20-22) to be constructed from the changes in spectrum area (normalised by gate time). 


\section{Photostability:}

Samples were irradiated continuously by the $450 \mathrm{~W}$ Xenon lamp of an Edinburgh Instruments FLS920 spectrometer, monochromated on the $\mathrm{S}_{0^{-0} 0}$ electronic band of each material (526 nm for PDIref, and $552 \mathrm{~nm}$ for PDIs1-3) and with 2nm bandpass. The emission intensity was continuously recorded over an hour using the detection arm of the same instrument, collected in kinetics mode.

\section{Transient absorption spectroscopy:}

The laser is PHAROS from Light Conversion (wavelength: $1030 \mathrm{~nm}$, pulse duration: $180 \mathrm{fs}$, repetition rate: $10 \mathrm{KHz}$, power: $6 \mathrm{~W}$ ). Part of the output is used to do third harmonic generation (THG), which produces $343 \mathrm{~nm}$ output. We use this $343 \mathrm{~nm}$ output to pump our samples and the power used is about $8 \mathrm{~mW}$. Another part of the output is used to pump a 2 mm sapphire plate to generate white light continuum (WLC). We use this WLC to probe the dynamics of the excited states. The polarization of the pump is vertical to the optical table, while the polarization of the probe is parallel to the optical table. The spot sizes (FW@1/e2) of the pump and probe are $360 \mu \mathrm{m}$ and $150 \mu \mathrm{m}$, respectively. The time delay between pump and probe is controlled by a 1 meter motorized translation stage (Zaber Technologies Inc. ALST1000AKT07G06SU), which can generate about $6 \mathrm{~ns}$ delay range. The pump is modulated by an optical chopper (Thorlabs Inc. MC2000B-EC) that is locked to the half frequency of the laser repetition rate to create an iterated pump on / pump off situation. The WLC goes through a spectrometer and the intensities at different wavelengths are monitored by a camera (Imaging Solutions Group LightWise LW-ELIS-1024A-1394). The camera is synchronized with the laser pulse to ensure it captures the spectrum of WLC pulse by pulse. The transient absorption spectrum at one fixed time delay is calculated by

$$
\Delta T / T=\left\{\sum_{i=1}^{N} \frac{\text { Spectrum_pump_on }(i)-S p e c t r u m \_p u m p \_o f f(i)}{\text { Spectrum_pump_off }(i)}\right\} / N
$$

where $\mathrm{N}$ is average number, in our case $\mathrm{N}=5120$. By changing the time delay between pump and probe, we obtain the whole transient absorption spectra. 
Reaction scheme of perylene derivatives:
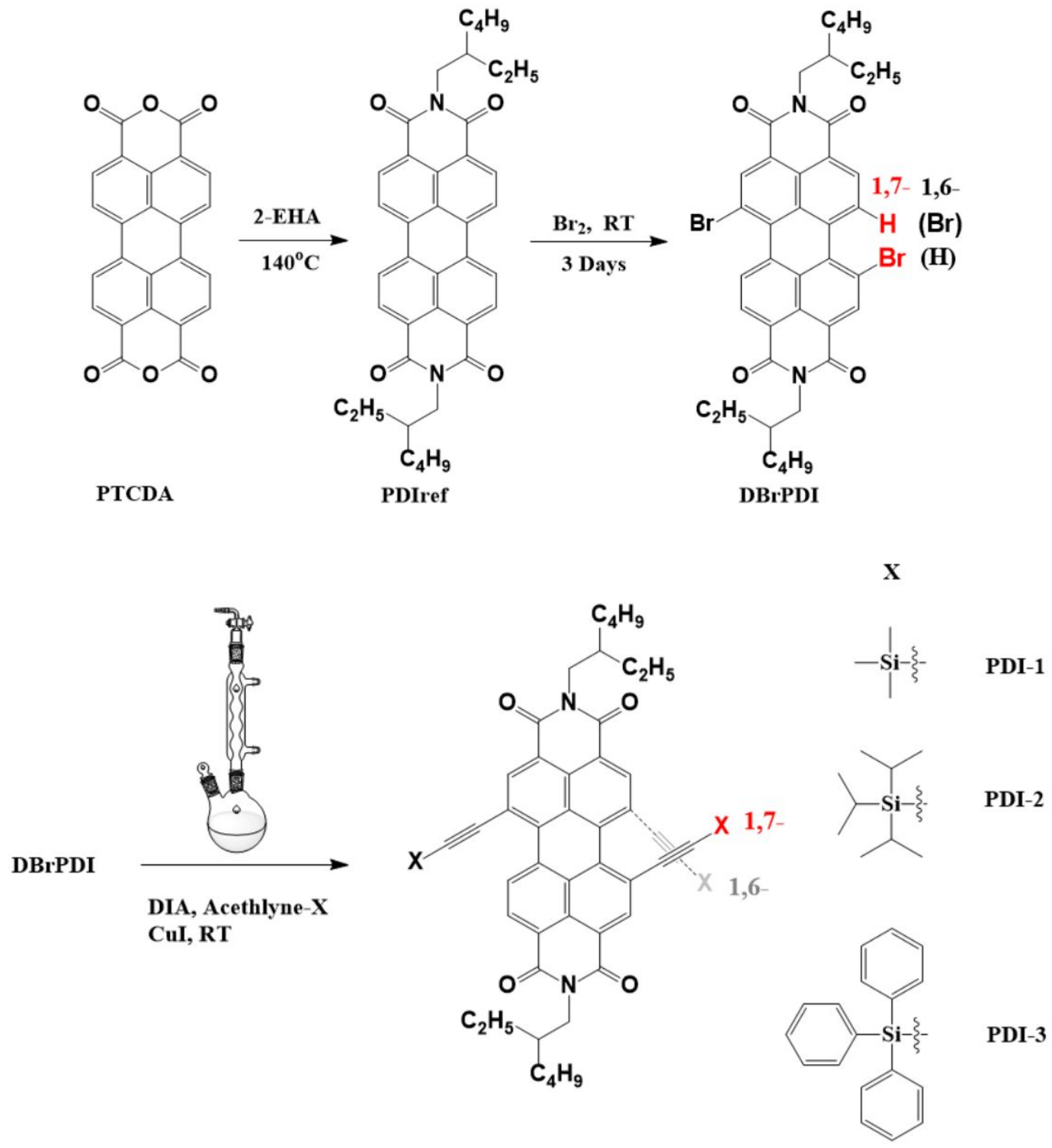

Figure S1: Reaction scheme of PDIref and PDI, 2 and 3. 


\section{Synthesis of N, N'-di-(2-ethylhexyl)-Perylene diimide (PDIref):}

PTCDA (3.92 grams, $10 \mathrm{mmol})$, DMF (100 mL) and 2-Ethyl-1-hexylamine (13 mL, $79 \mathrm{mmol})$ were added to the $250 \mathrm{~mL}$ 2-neck flask. The mixture was stirred under reflux for 30 minutes at $60^{\circ} \mathrm{C}$ and then at 140 degrees for 24 hours under reflux. Then, the mixture was cooled to room temperature and transferred into a $1 \mathrm{M} 500 \mathrm{~mL} \mathrm{HCl}_{(\mathrm{aq})}$ solution. The mixture was stirred overnight, then filtered in vacuum with nuche filter and washed with hot $\mathrm{NaOH}_{(\mathrm{aq})}$ solution. Finally, it was washed with plenty of hot distilled water. The solid material was dried in the oven, then purified by column chromatography $\left(\mathrm{SiO}_{2}: \mathrm{CHCl}_{3}\right) .{ }^{1} \mathbf{H}$ NMR (400 $\mathbf{M H z}$, Chloroform-d, TMS/ppm) $\boldsymbol{\delta}:$ 8.59-8.57, 8.48-8.46 (d, d J: 8Hz, 8H-perylene core $\left.\mathrm{C}_{\mathrm{Ar}} \mathrm{H}\right)$, $4.13(\mathrm{~m}, 4 \mathrm{H}), 1.96(\mathrm{~m}, 2 \mathrm{H}), 1.4(\mathrm{~m}, 13 \mathrm{H}), 0.96-0.90(\mathrm{~m}, \mathrm{~m}, 12 \mathrm{H})$.

\section{Synthesis of N, N'-di-(2-ethylhexyl)-1,7 (6)-dibromo-Perylene diimide (DBrPDI):}

PDIref $(2 \mathrm{~g})$ and chloroform $(120 \mathrm{~mL})$ were added into two necked flasks. After the mixture was stirred for 10 minutes, bromine $(20 \mathrm{~mL})$ was added slowly. It was stirred under reflux at $50^{\circ} \mathrm{C}$ for 3 hours. Sodium Bisulfite solution was added slowly to terminate the reaction. After extraction with chloroform, the organic phase evaporated. It was then purified by column chromatography $\left(\mathrm{SiO}_{2}: \mathrm{CHCl}_{3}\right)$ [Yield: $\sim 76 \%$ (1,6 and 1,7-Brominated)]. ${ }^{\mathbf{1}} \mathbf{H}$ NMR $\mathbf{d B r P D I}_{\mathbf{d}}$ (400 MHz, Chloroform-d, TMS/ppm) $\boldsymbol{\delta}:$ 9.43-9.31 (m, 2H C $\left.\mathrm{Ar}_{\mathrm{rr}} \mathrm{H}\right), 8.84\left(\mathrm{~S}, 1 \mathrm{H} \mathrm{C}_{\mathrm{Ar}}-\mathrm{H}\right), 8.63-8.59$ (m, 3H C $\mathrm{Ar}-\mathrm{H}), 4.13\left(\mathrm{~m}, 4 \mathrm{H} \mathrm{N}-\mathrm{C}_{\text {aliphatic }}-\mathrm{H}\right), 1.94$ (m, 2H C aliphatic $\left.-\mathrm{H}\right), 1.40-1.31$ (m, 16H), 1.95, $1.90(\mathrm{t}, \mathrm{t}, 6 \mathrm{H}+6 \mathrm{H})$.

Synthesis of N, N'-di-(2-ethylhexyl)-1,7(6)-di(tri-methylsilyl)ethynylen-Perylene diimide (PDI1):

DiBrPDI $(0.77 \mathrm{~g}, 1 \mathrm{mmol})$ and diisopropylamine $(15 \mathrm{~mL})$ were added into a single neck flask, stirred for 15 minutes in an ultrasonic mixer under continous nitrogen gas purging. Tetrakis(triphenylphosphine)palladium $(0)\left[\mathrm{Pd}\left(\mathrm{PPh}_{3}\right)_{4}\right](115 \mathrm{mg}, 0.1 \mathrm{mmol})$ and copper(I)iodide (CuI) $(19 \mathrm{mg}, 0,1 \mathrm{mmol})$ were added into the mixture and then stirred for a further 15 minutes. Finally, trimethylsilyacetylene $(1.38 \mathrm{~mL}, 10 \mathrm{mmol})$ was added and stirred under dark conditions for 3 days at room temperature. The reaction mixture was poured into chloroform and extracted with water. Then, the organic phase was evaporated and the solid was purified by column chromatography (Silica gel, chloroform:hexane 2:1). [Yield: 84\% (1,6 and 1,7func.)] (1,7 pure isomers of PDI1 was purified with chloroform:hexane (1:1). ${ }^{1} \mathrm{H}$ NMR (400 MHz, Chloroform-d, TMS/ppm) $\delta$ : 10.13-10.10 (d, J:12 Hz, 2H), 8.74 (s, 2H), 8.558.53 (d, J:8 Hz, 2H), 7.25 (Solvent-CDCl $\left.{ }_{3}\right), 4.15$ (m, 4H), 1.94 (m, 2H), 1.42-1.32 (m, 16H), 
$0.95(\mathrm{t}, 6 \mathrm{H}), 0.90(\mathrm{t}, 6 \mathrm{H})$. MALDI-TOF MS, Found: $[\mathrm{M}+\mathrm{H}]^{+} \mathrm{m} / \mathrm{z}$ : 808.6; molecular weight: $807.18 \mathrm{~g} / \mathrm{mol}$.

\section{Synthesis of N, N'-di-(2-ethylhexyl)-1,7(6)-di(triisopropylsilyl)ethynylen-Perylene diimide (PDI2):}

It was synthesized in the same manner as the synthesis of PDI1 above. $2.25 \mathrm{~mL}(10 \mathrm{mmol})$ of triisopropylsilyacetylene was used. The solid was purified by column chromatography (Silica gel, chloroform:hexane 2:1) [Yield: $\sim 93 \%$ (1,6 and 1,7-func.)]. Isomeric purity N,N'-di-(2ethylhexyl)-1,7-di(triisopropylsilyl)ethynylen-perylene diimide (1,7-PDI2) and N,N'-di-(2ethylhexyl)-1,6-di(triisopropylsilyl)ethynylen-perylene diimide (1,6-PDI2) were purified in by column cormatography $\left(\mathrm{SiO}_{2}\right)$ using the chloroform:hexane $(1: 1)$ mobile phase. ${ }^{1} \mathbf{H}-\mathbf{N M R}$ (1,7-regioisomerical pure PDI2) (400 MHz, Chloroform-d, TMS/ppm) 8: 10.33-10.31 (d, $\mathrm{J}: 8 \mathrm{~Hz}, 2 \mathrm{H}), 8.81$ (s, 2H), 8.60-8.58 (d, J:8 Hz, 2H), 4.15 (m, 4H), 1.97(m, 2H), 1.42-1.22 (m, overlap, 64H total), 0.96 (t, J:8Hz, 6H), 0.89 (t, J:8H, 6H). ${ }^{13}$ C-NMR (1,7-regioisomerical

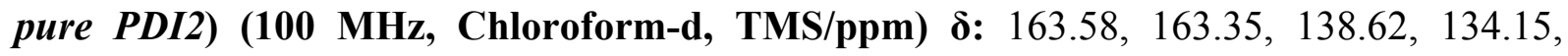
130.67, 127.71, 127.48, 123.10, 122.02, 120.08, 107.51, 103.88, 44.43, 38.02, 30.81, 28.76, 24.12, 23.08, 18.80, 14.10, 11.36, 10.64. 1H-NMR (1,6-regioisomerical pure PDI2) (400 MHz, Chloroform-d, TMS/ppm) $\delta: 10.37-10.35$ (d, J:8Hz, 2H), 8.83(s, 2H), 8.67-8.65 (d, $\mathrm{J}: 8 \mathrm{~Hz}, 2 \mathrm{H}), 4.19(\mathrm{~m}, 4 \mathrm{H}), 1.9(\mathrm{~m}, 2 \mathrm{H}), 1.32-1.21$ (m, overlap, 64H total), 0.97 (triplet of doublets, 6H), 0.90 (triplet of doublets, 6H). ${ }^{13}$ C-NMR (1,6-regioisomerical pure PDI2) (100 MHz, Chloroform-d, TMS/ppm) $\delta$ : 163.84, 133.63, 130.41, 127.20, 123.52, 121.69, 120.74, $107.61,103.67,38.07,30.81,28.72,24.16,23.06,18.80,14.11,11.38,10.66$.

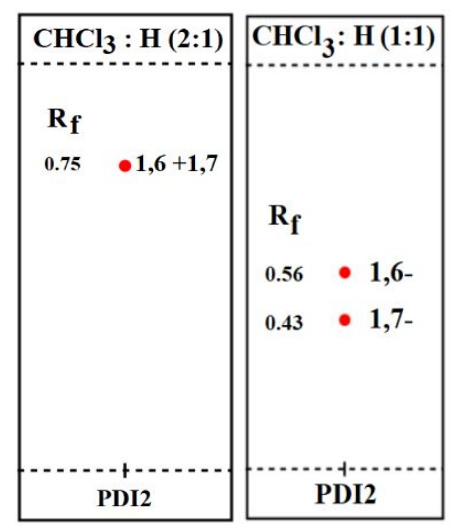

Figure S2: Thin layer chromatography of mobile phases of 1.6 and 1.7 functional PDI2 in 2 different polarities. The TLC on the left shows that isomeric mixtures are not separated, while on the right, 1.6 and 1.7 mixtures of isomeric purity are separated. 
Synthesis of N, N'-di-(2-ethylhexyl)-1,7(6)-di(triphenylsilyl)ethynylen-Perylene diimide (PDI3):

It was synthesized in the same manner as the synthesis of PDI1 or PDI2 above. $2.88 \mathrm{~g}$ (10mmol) of triphenylsilyacetylene was used. The solid was purified by column chromatography (Silica gel, chloroform:hexane 1:1 to 2:1) [Yield: $55 \%$ (1,6 and 1,7-func.)]. Obtained as regiaisomerical mix 1,6 + 1,7 PDI3. ${ }^{1} \mathbf{H}$ NMR (400 MHz, Chloroform-d, TMS/ppm) $\boldsymbol{\delta}: 10.03$ (1,7 and 1,6-regioisomericalmix, dd, 2H), 8.97-8.93 (dd, 2H), 8.26-8.23 (dd, 2H), 7.76-7.42 (m, overlap, 30H total), 4.17 (m, 4H), 1.99 (m, 2H), 1.47-1.23 (m, overlap, $16 \mathrm{H}), 0.98(\mathrm{t}, 6 \mathrm{H}), 0.92(\mathrm{t}, 6 \mathrm{H})$. The 1.6 and 1.7 regio isomers of PDI3 could not be purified. used as a mixture. MALDI-TOF MS, Found: $[\mathrm{M}+\mathrm{H}]^{+} \mathrm{m} / \mathrm{z}$ : 1180.8; molecular weight: $1179.59 \mathrm{~g} / \mathrm{mol}$.

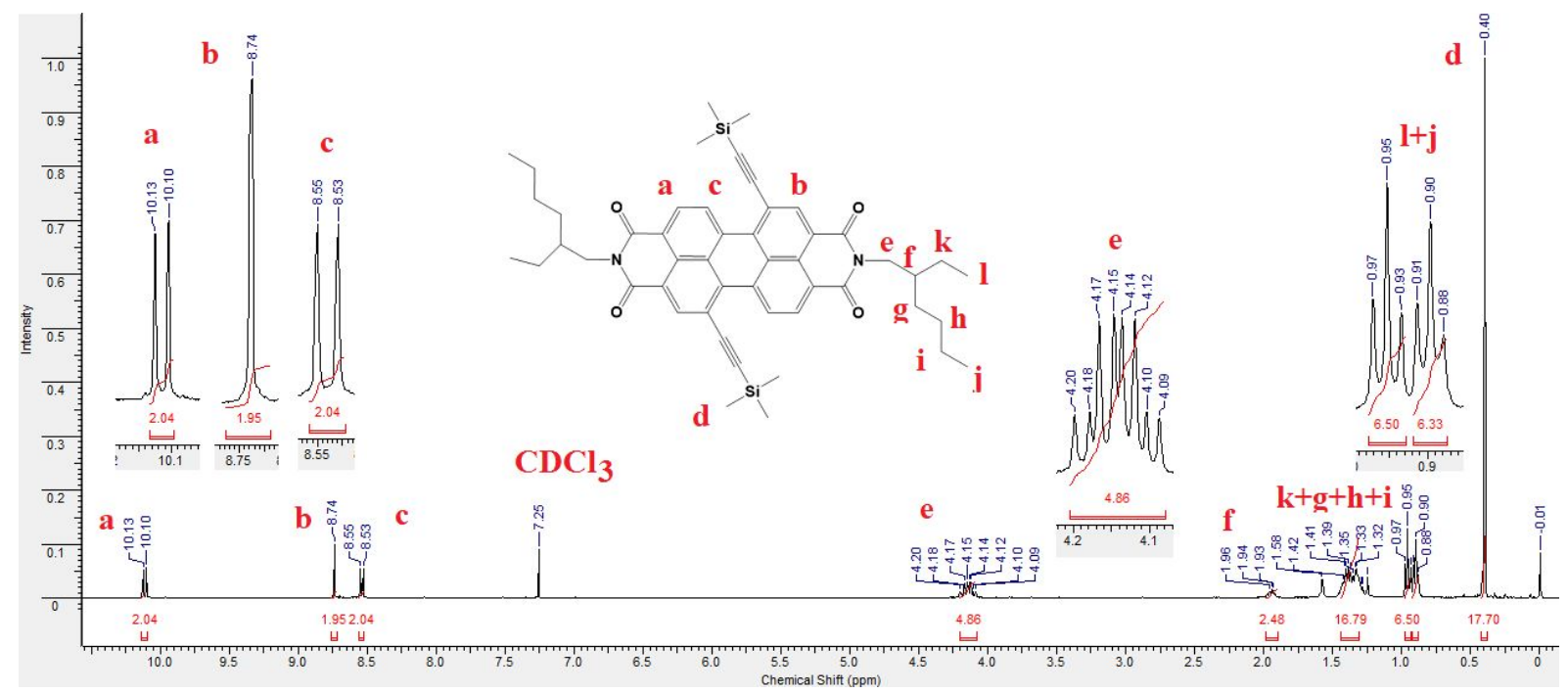

Figure S3: ${ }^{1} \mathrm{H}-\mathrm{NMR}$ of 1,7-regioisomerical pure PDI1 


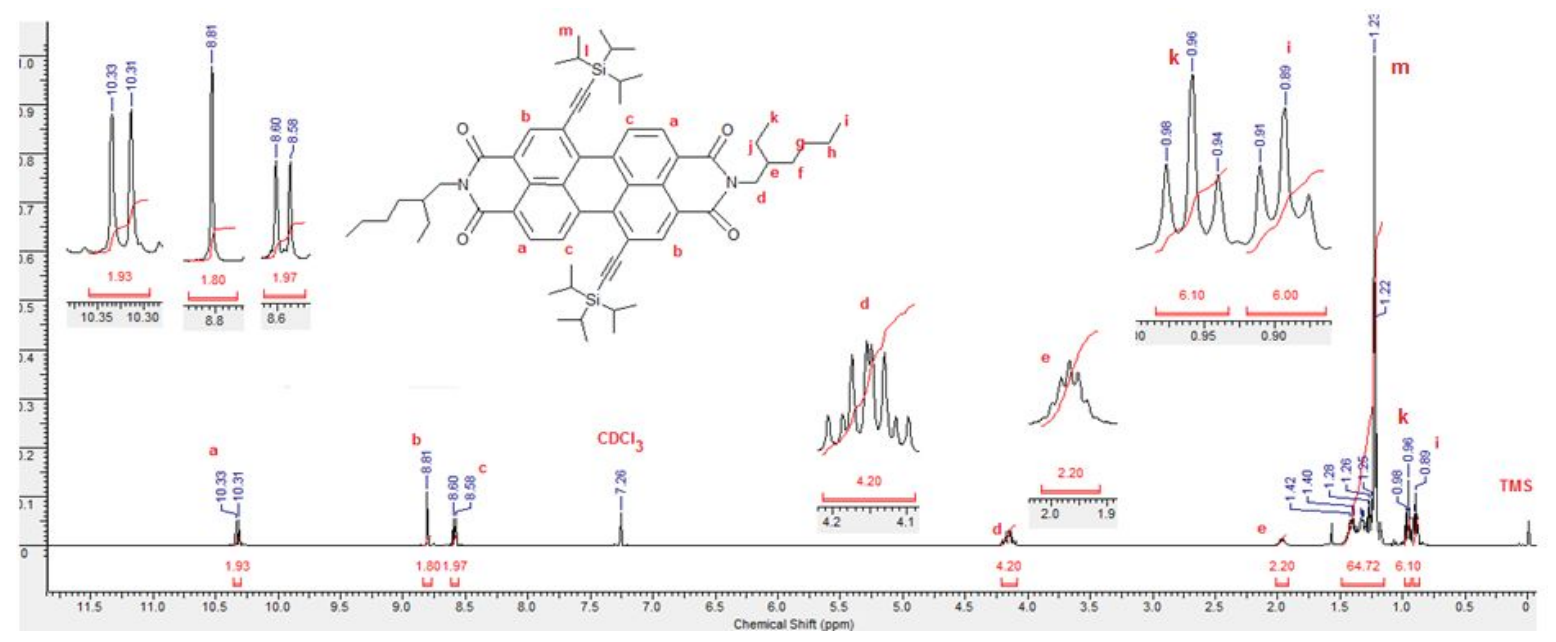

Figure S4: ${ }^{1} \mathrm{H}-\mathrm{NMR}$ of 1,7-regioisomerical pure PDI2

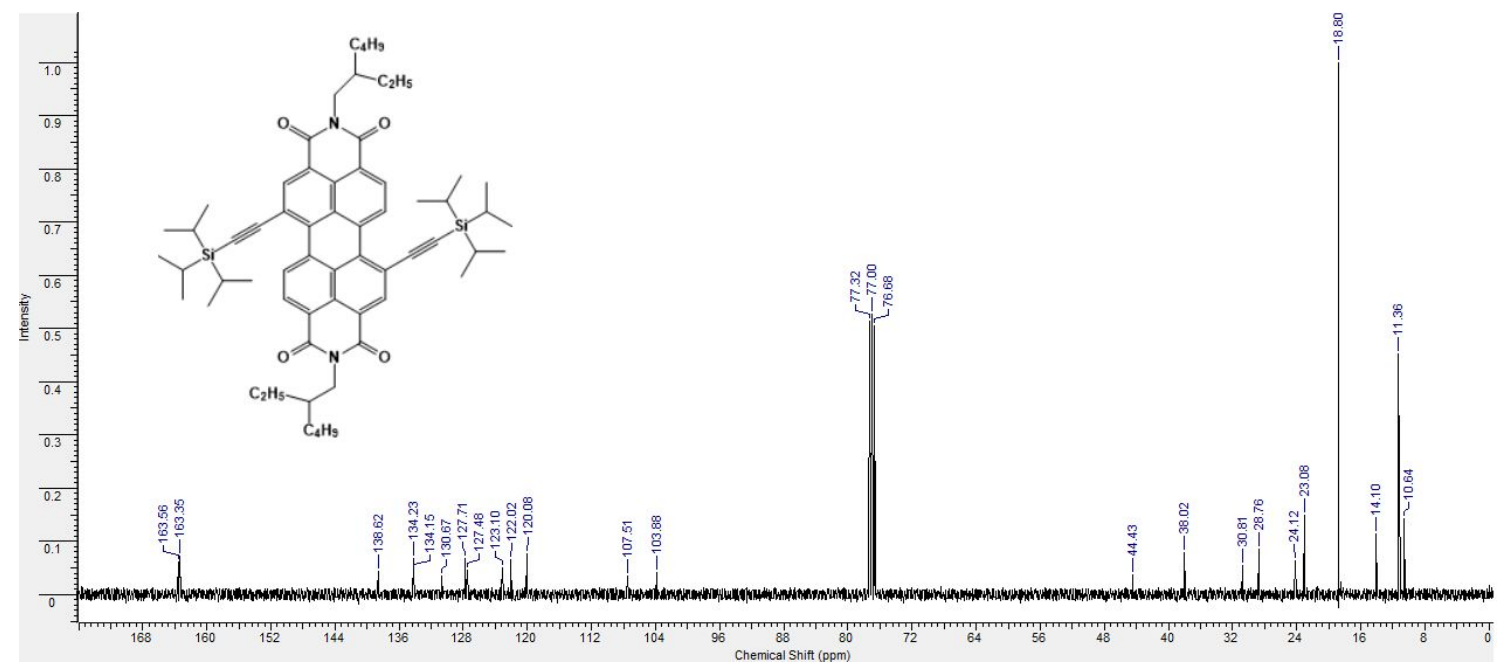

Figure S5: ${ }^{13} \mathrm{C}-\mathrm{NMR}$ of 1,7-regioisomerical pure PDI2 


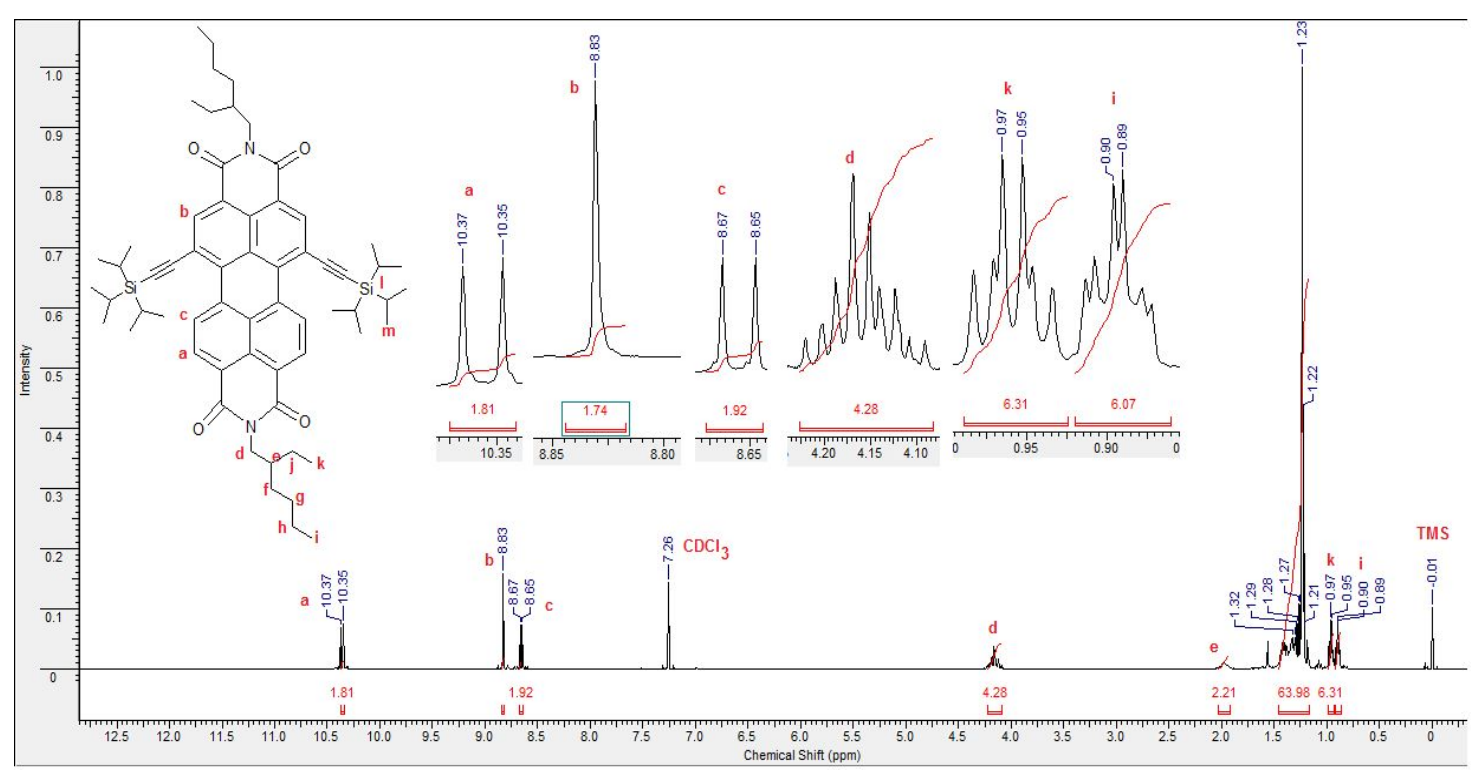

Figure S6: ${ }^{1} \mathrm{H}-\mathrm{NMR}$ of 1,6-regioisomerical pure PDI2

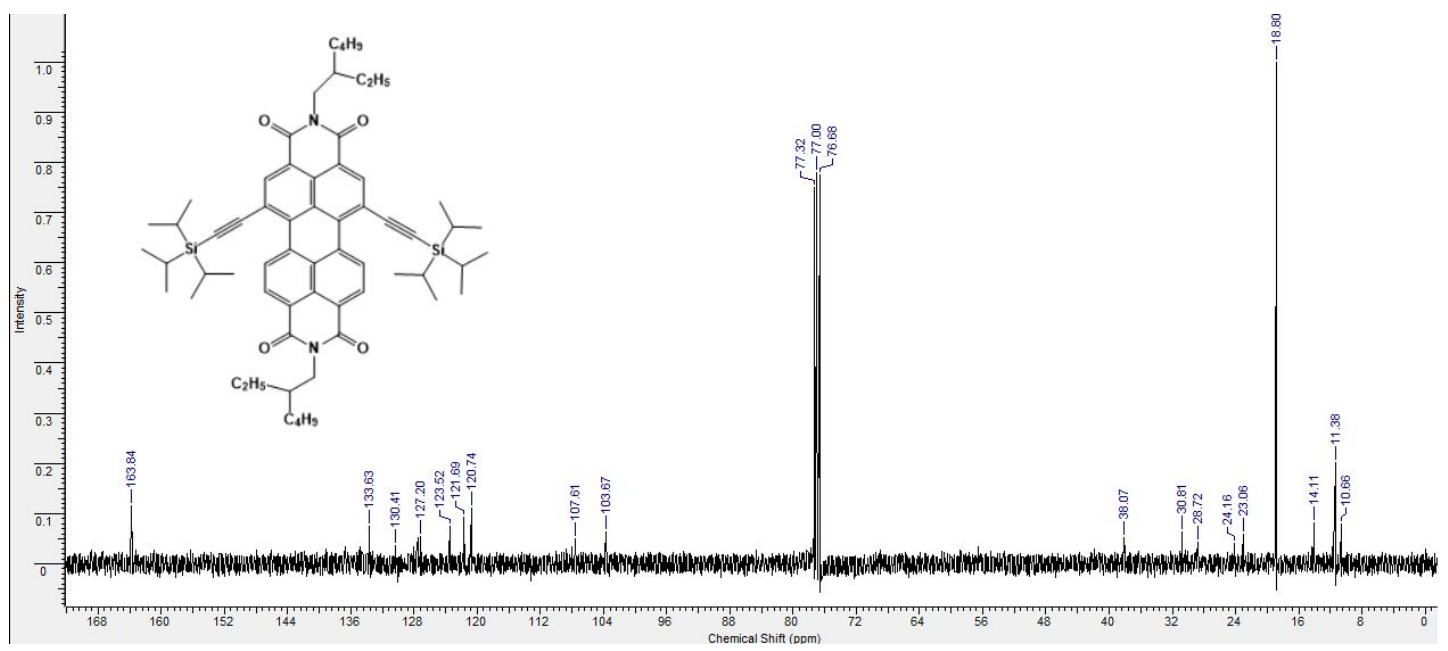

Figure S7: ${ }^{13} \mathrm{C}$-NMR of 1,6-regioisomerical pure PDI2 


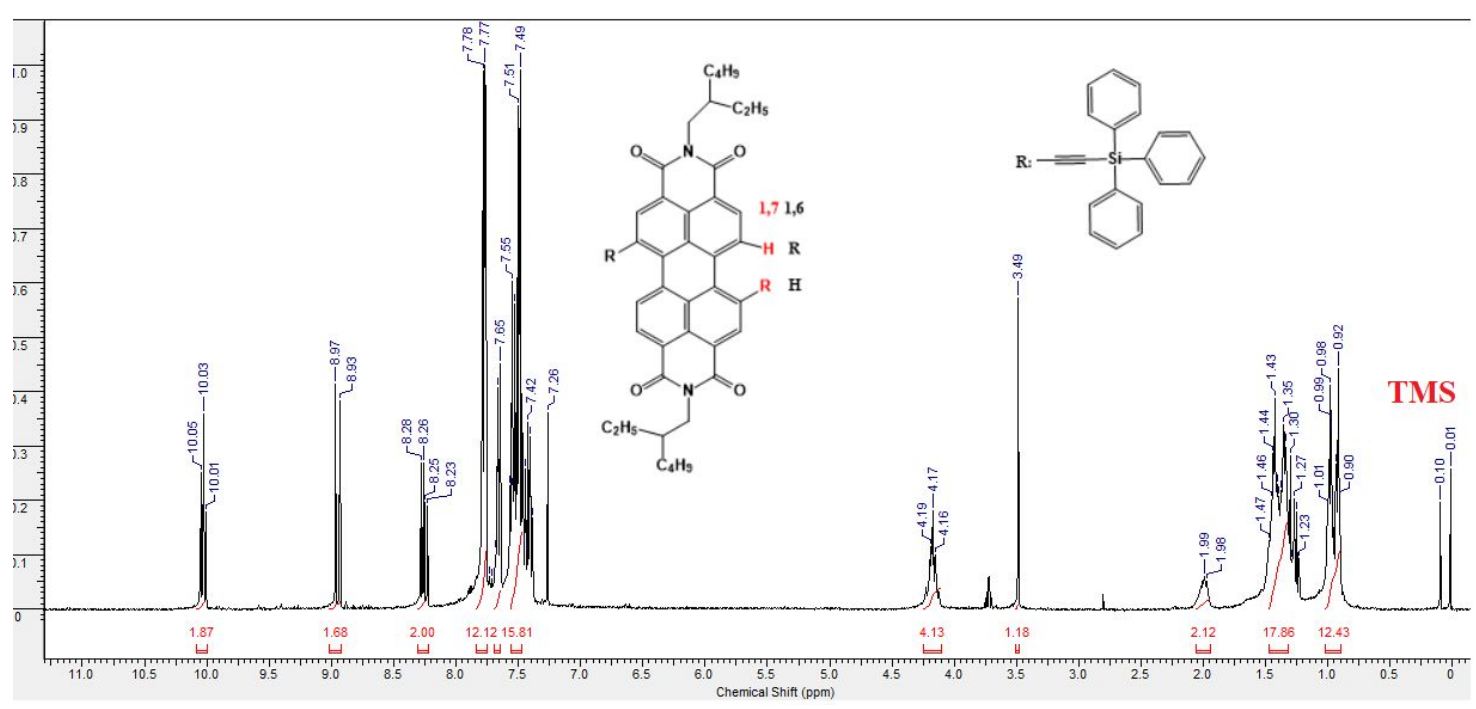

Figure S8: ${ }^{1} \mathrm{H}-\mathrm{NMR}$ spectrum of 1,7 (6)-PDI3

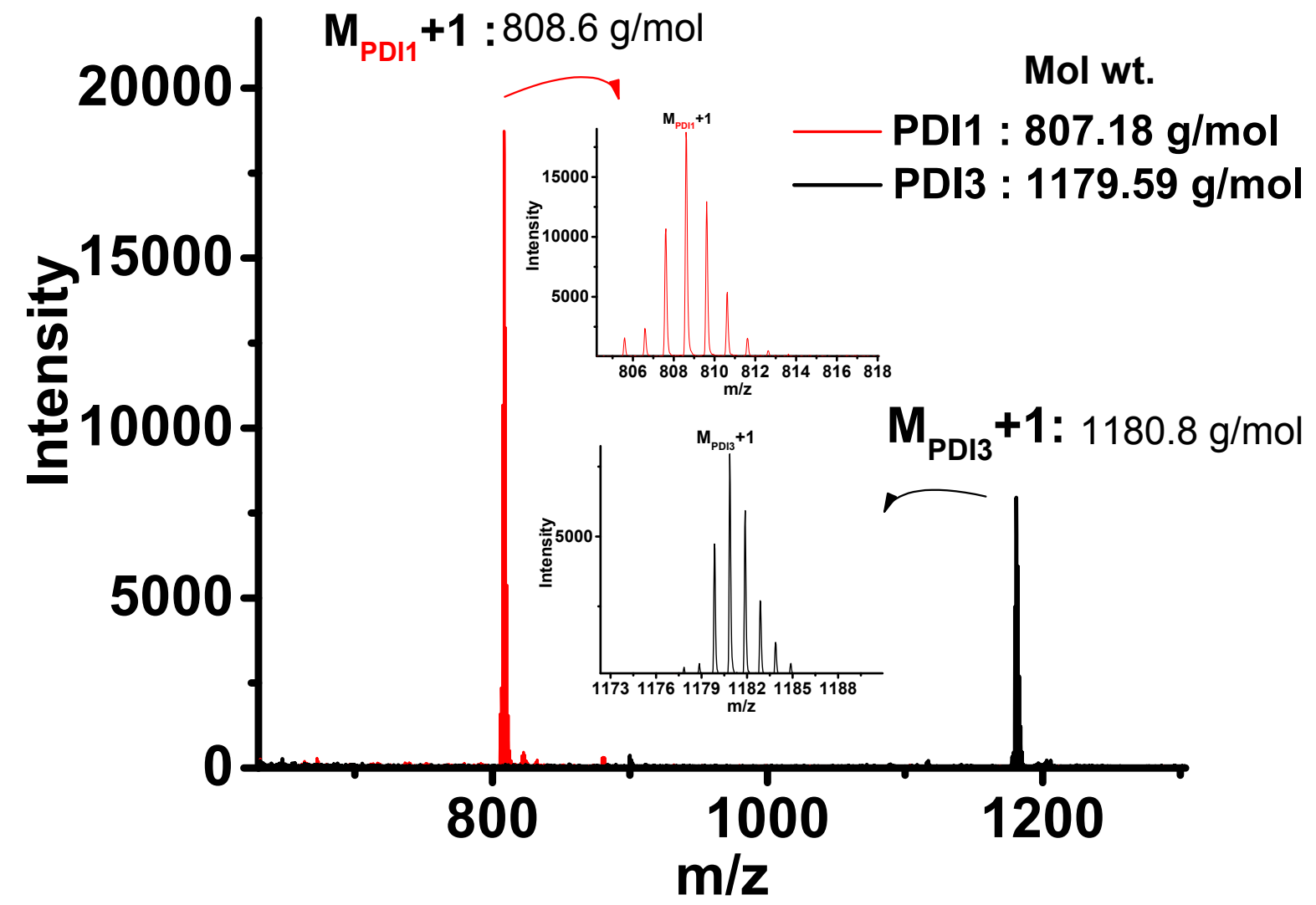

Figure S9: HRMS (MALDI-TOF) of PDI1 and PDI3. LC/MS data for PDI2 previously reported - referred to as PDI1 in that work. ${ }^{6}$ 


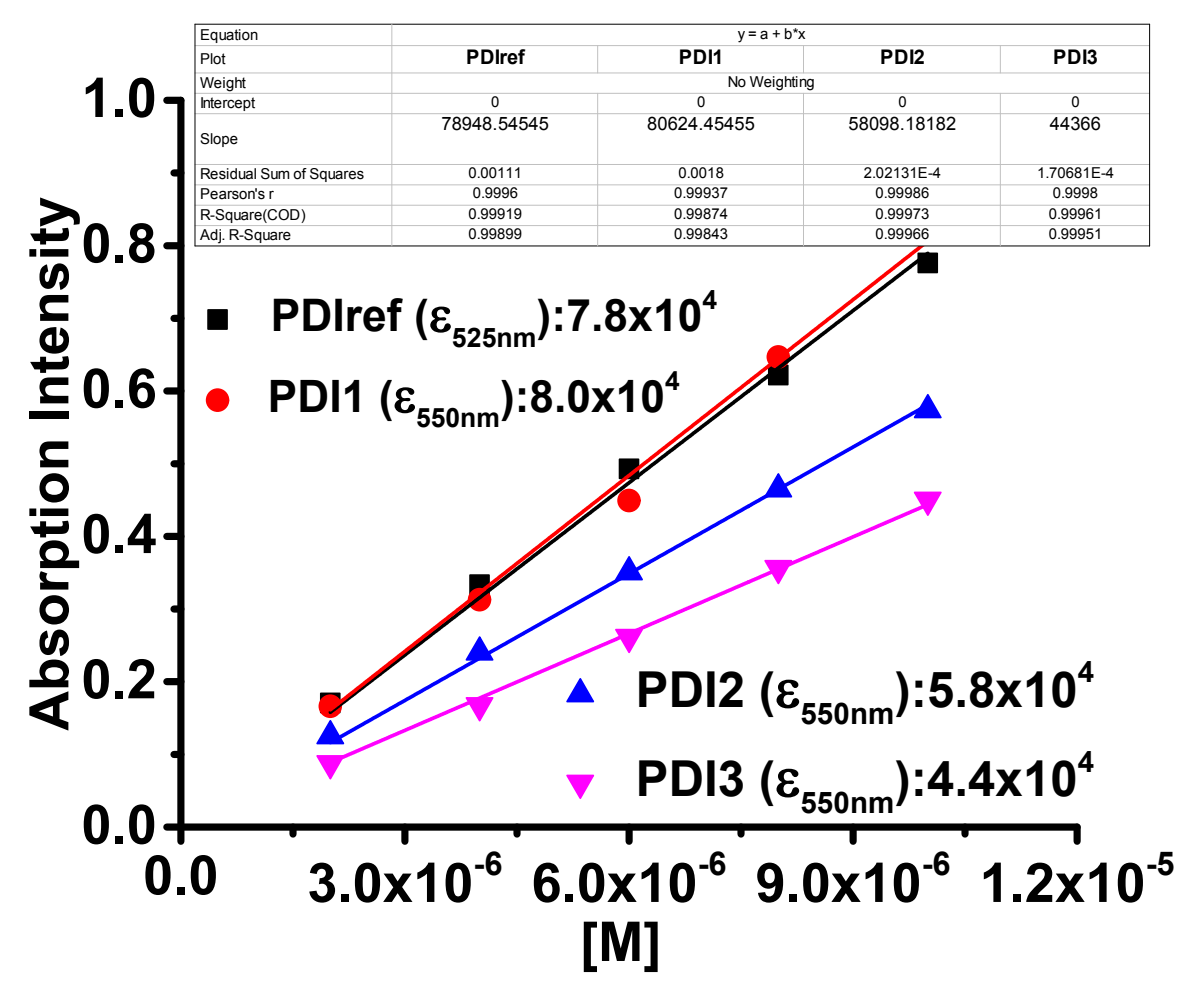

Figure S10. Molar extinction coefficients of PDIref and PDIs1-3 on main absorbance peak in chloroform solution.

Table S1: Electrochemical and Energy levels properties of PDIs.

\begin{tabular}{|c|c|c|c|c|c|}
\hline PDIs & $\begin{array}{c}\text { First } \\
\text { reductive } \\
\text { half-wave } \\
\text { potential (V) }\end{array}$ & $\begin{array}{c}\mathrm{E}_{\text {oxd }} \\
(\mathrm{V})\end{array}$ & $\begin{array}{c}\Delta \mathrm{E}_{\text {opt }} \\
(\mathrm{eV})\end{array}$ & $\begin{array}{c}\text { LUMO } \\
(\mathrm{eV})\end{array}$ & $\begin{array}{c}\text { HOMO } \\
(\mathrm{eV})\end{array}$ \\
\hline PDIref & -0.61 & - & 2.28 & -3.69 & -5.97 \\
\hline PDI1 & -0.55 & - & 2.15 & -3.77 & -5.92 \\
\hline PDI2 & -0.58 & - & 2.15 & -3.74 & -5.89 \\
\hline PDI3 & -0.52 & - & 2.15 & -3.79 & -5.94 \\
\hline Ferrocene & - & 0.45 & & & \\
\hline
\end{tabular}



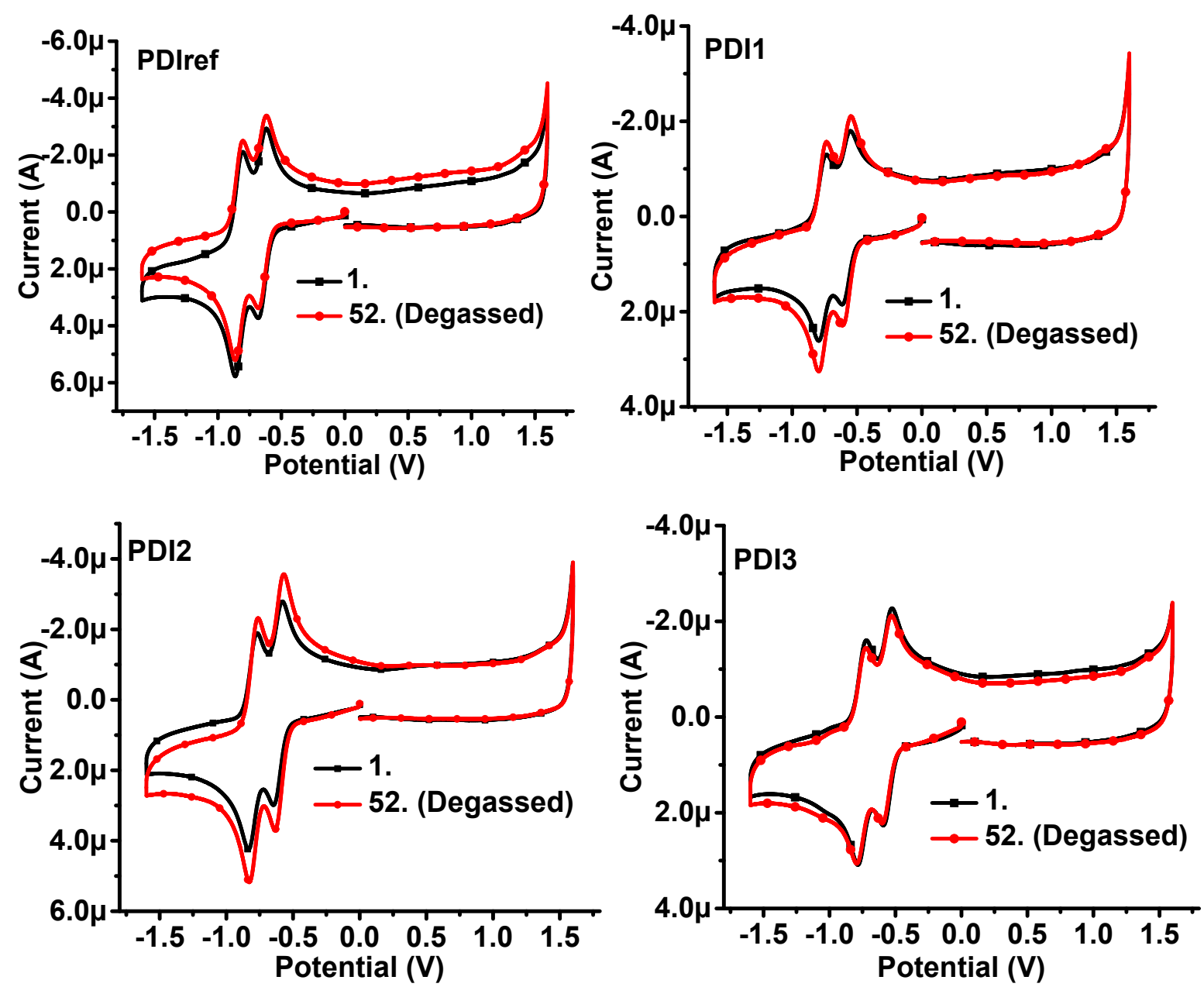

Figure S11. Electrochemical stability tests of PDIs. After 51 full round cycles were done, the oxygen peaks around $-1 \mathrm{~V}$ were degassed with nitrogen gas and the 52nd full cycle was compared with the first cycle.

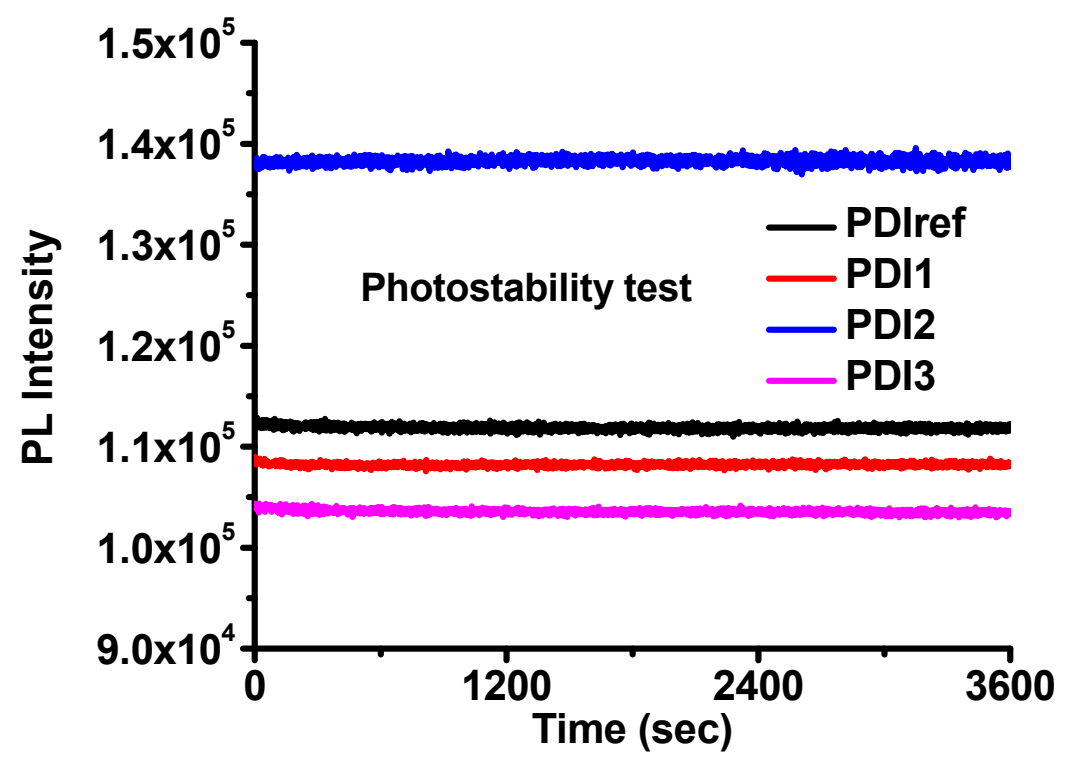

Figure S12. Photostability test of PDIref, PDI1, 2 and 3 in chloroform ( $\lambda_{\text {ext }}: \mathrm{S}_{0^{-0} 0}$ electronic). 

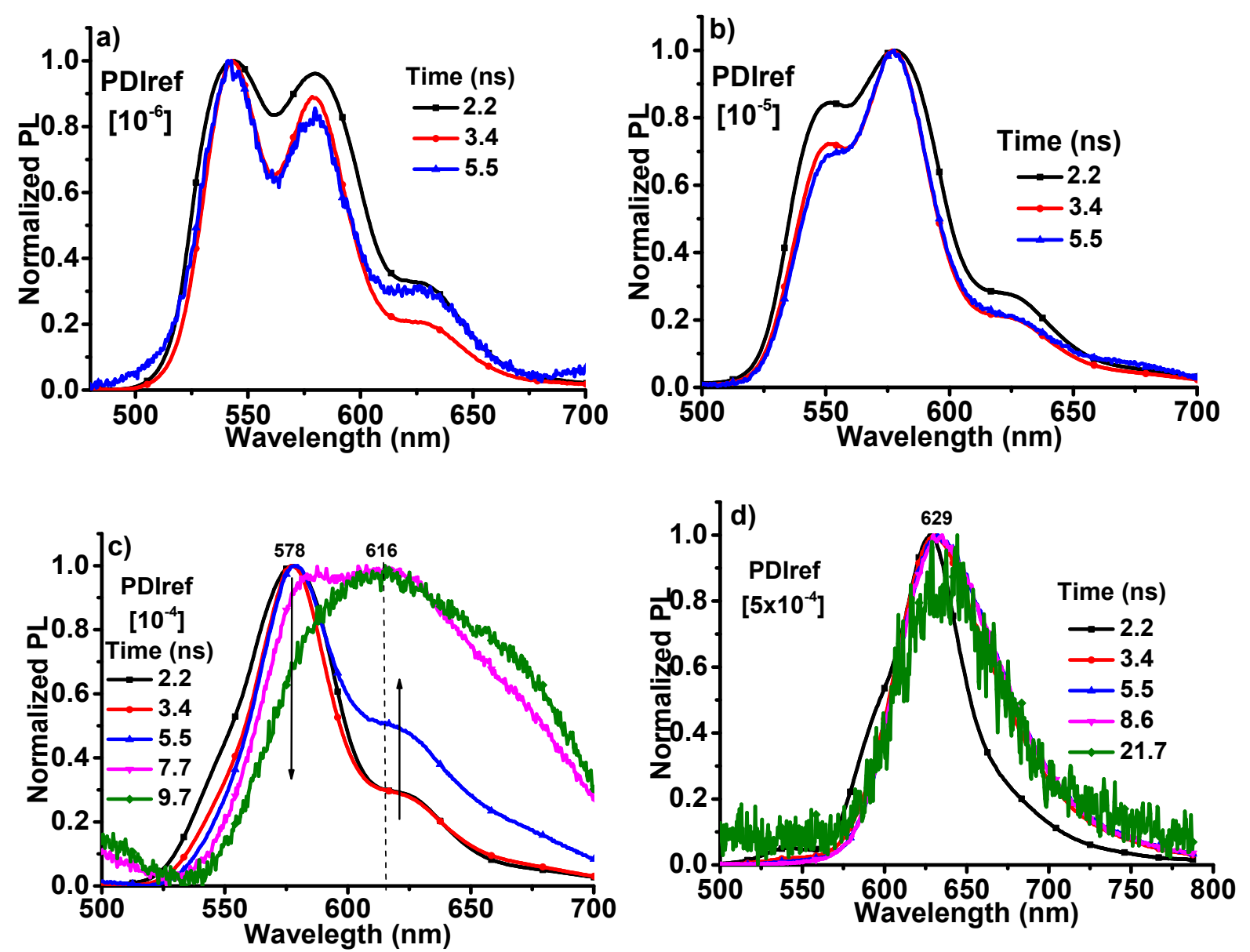

Figure S13: Time resolved PL spectra of PDIref at a) $10^{-6} \mathrm{M}$, b) $10^{-5} \mathrm{M}$, c) $10^{-4} \mathrm{M}$ and d) $5 \times 10^{-4}$ $\mathrm{M}$ in chloroform. 

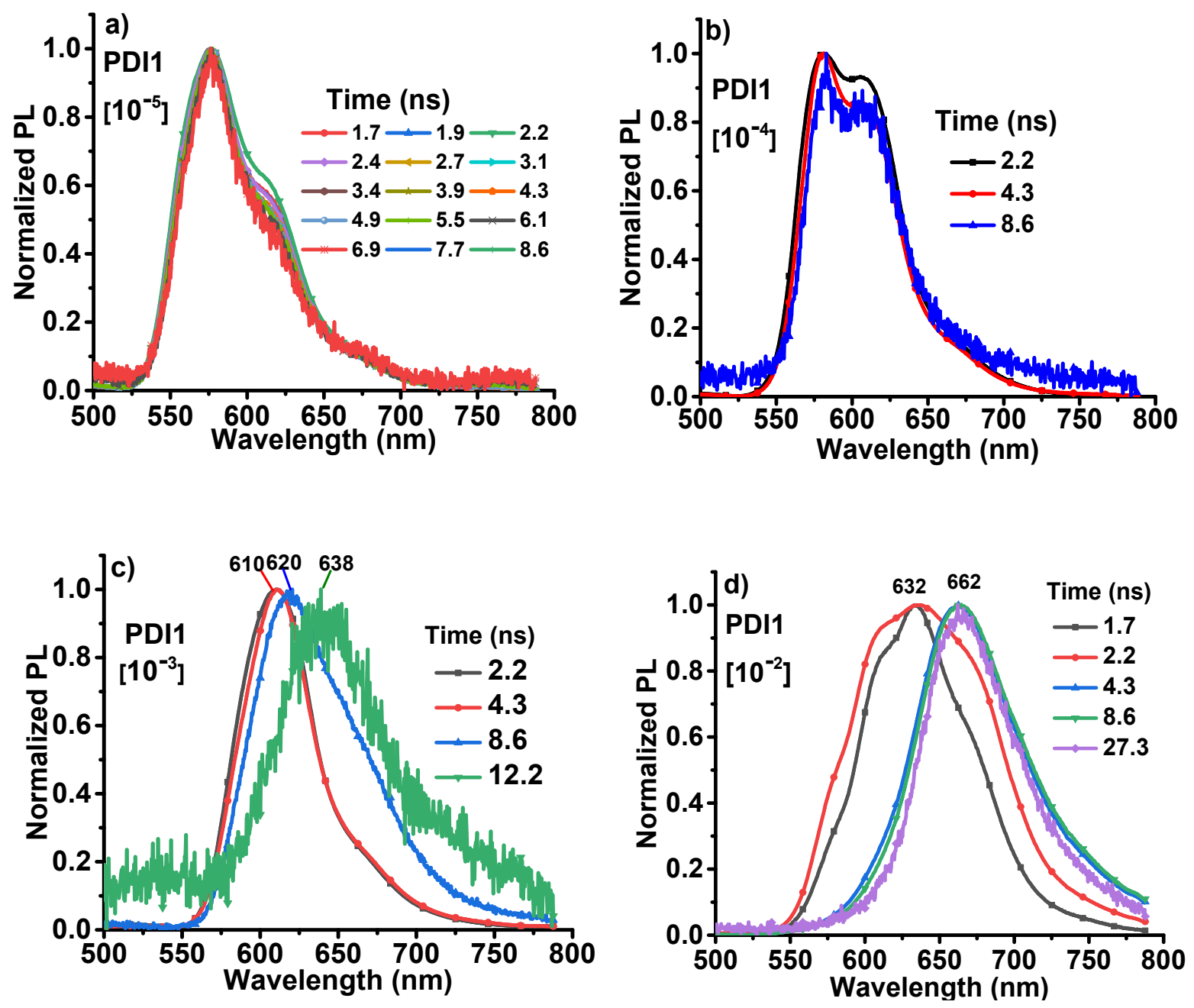

Figure S14: Time resolved PL spectra of PDI1 at a) $10^{-5} \mathrm{M}$, b) $10^{-4} \mathrm{M}$, c) $10^{-3} \mathrm{M}$ and d) $10^{-2} \mathrm{M}$ in chloroform. 

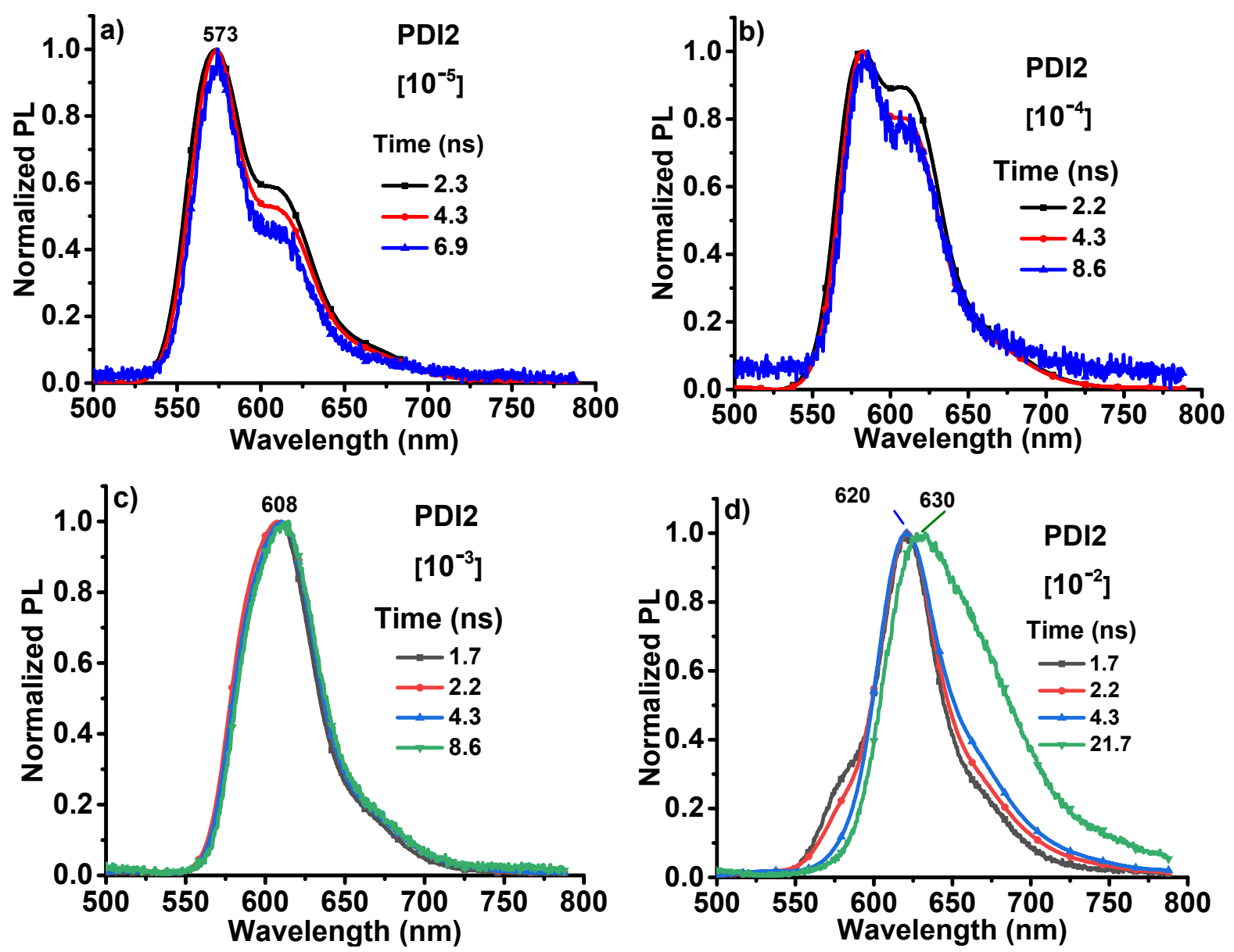

Figure S15: Time resolved PL spectra of PDI2 at a) $10^{-5} \mathrm{M}$, b) $10^{-4} \mathrm{M}$, c) $10^{-3} \mathrm{M}$ and d) $10^{-2} \mathrm{M}$ in chloroform. 

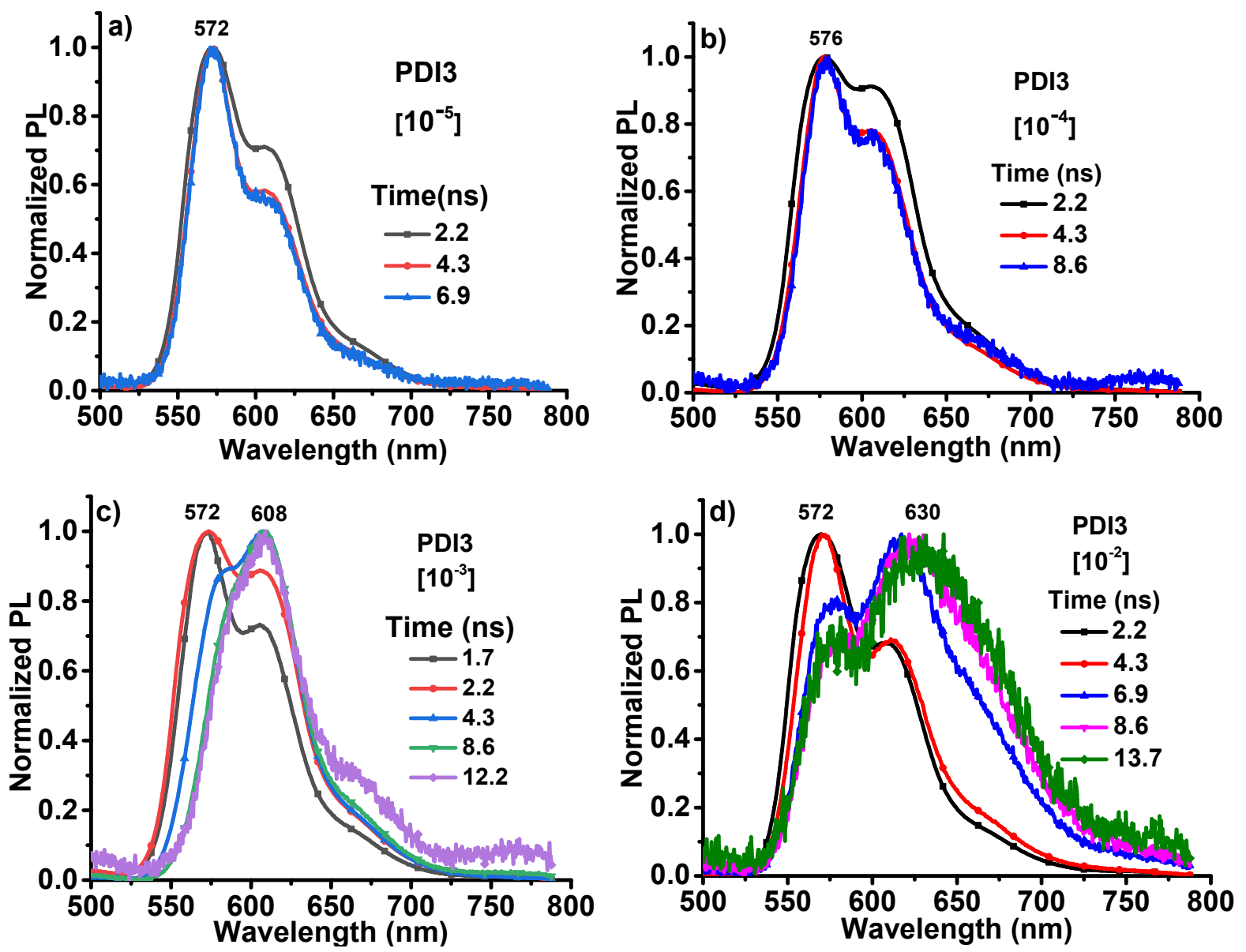

Figure S16: Time resolved PL spectra of PDI3 at a) $10^{-5} \mathrm{M}$, b) $10^{-4} \mathrm{M}$, c) $10^{-3} \mathrm{M}$ and d) $10^{-2} \mathrm{M}$ in chloroform.

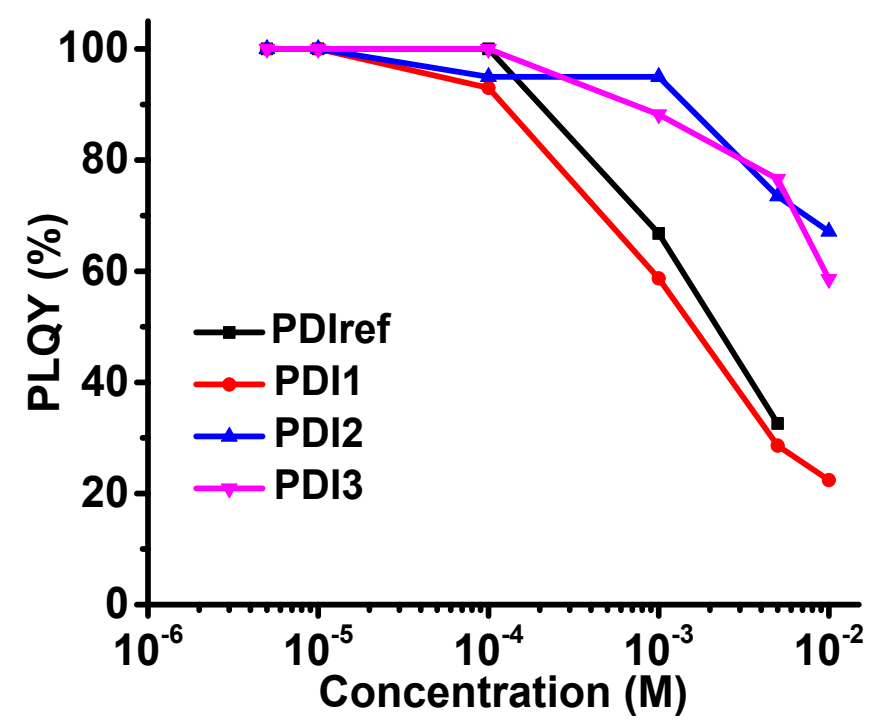

Figure S17: Photoluminescence quantum yields of PDIs at different concentrations in $\mathrm{CHCl}_{3}$ $\left(\lambda_{\text {ext }}: \mathrm{S}_{0^{-1} 1}\right.$ electronic band, i.e., $490 \mathrm{~nm}$ for PDIref and 512nm for PDIs1-3). 

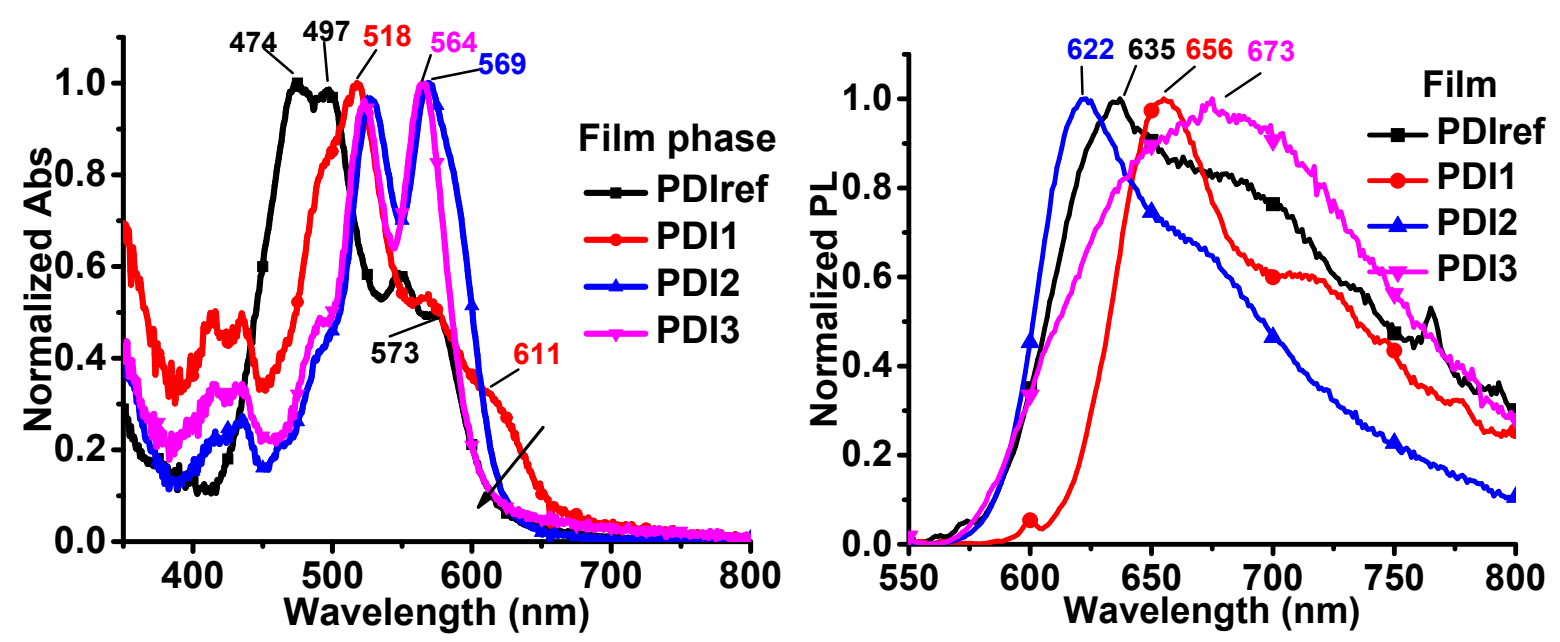

Figure S18: Normalized steady-state absorption and PL spectra of PDIref and PDIs 1-3 neat films.

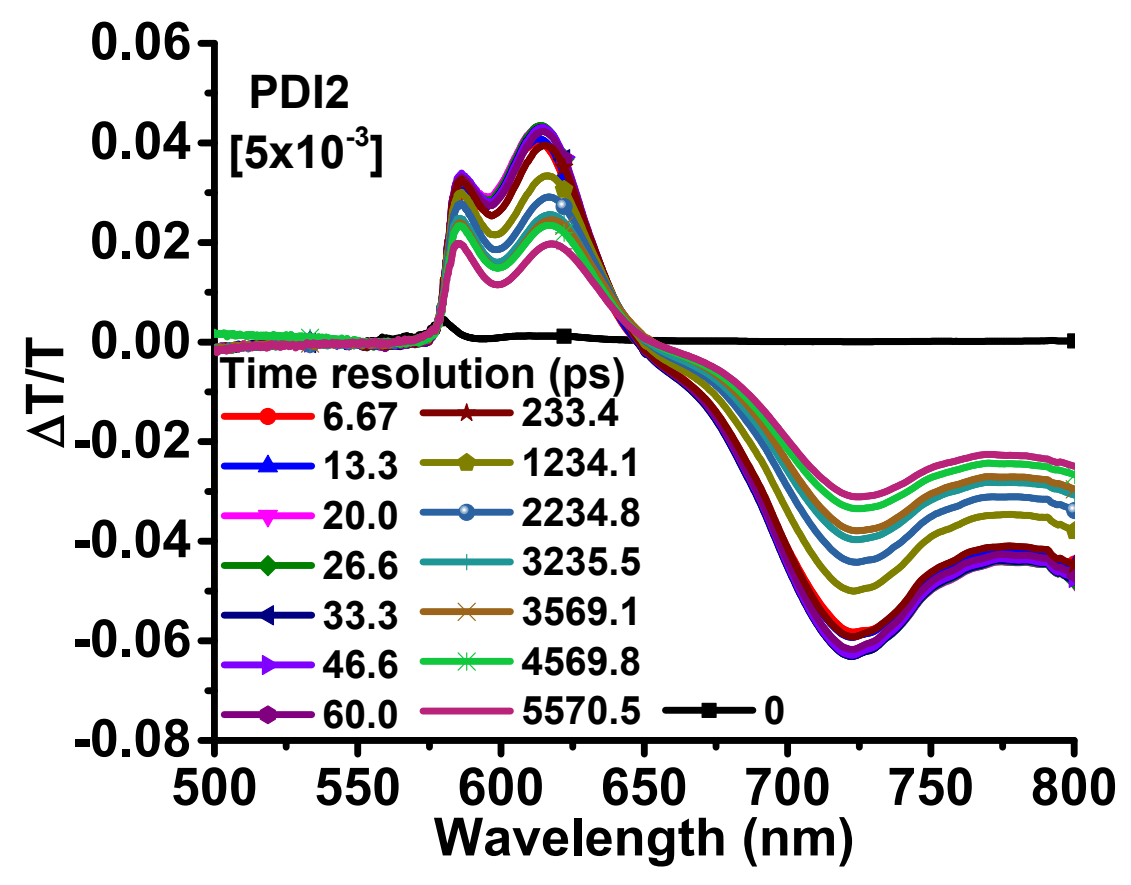

Figure S19: Example of negligible changes in TAS spectra at longer delay times for PDI2. 

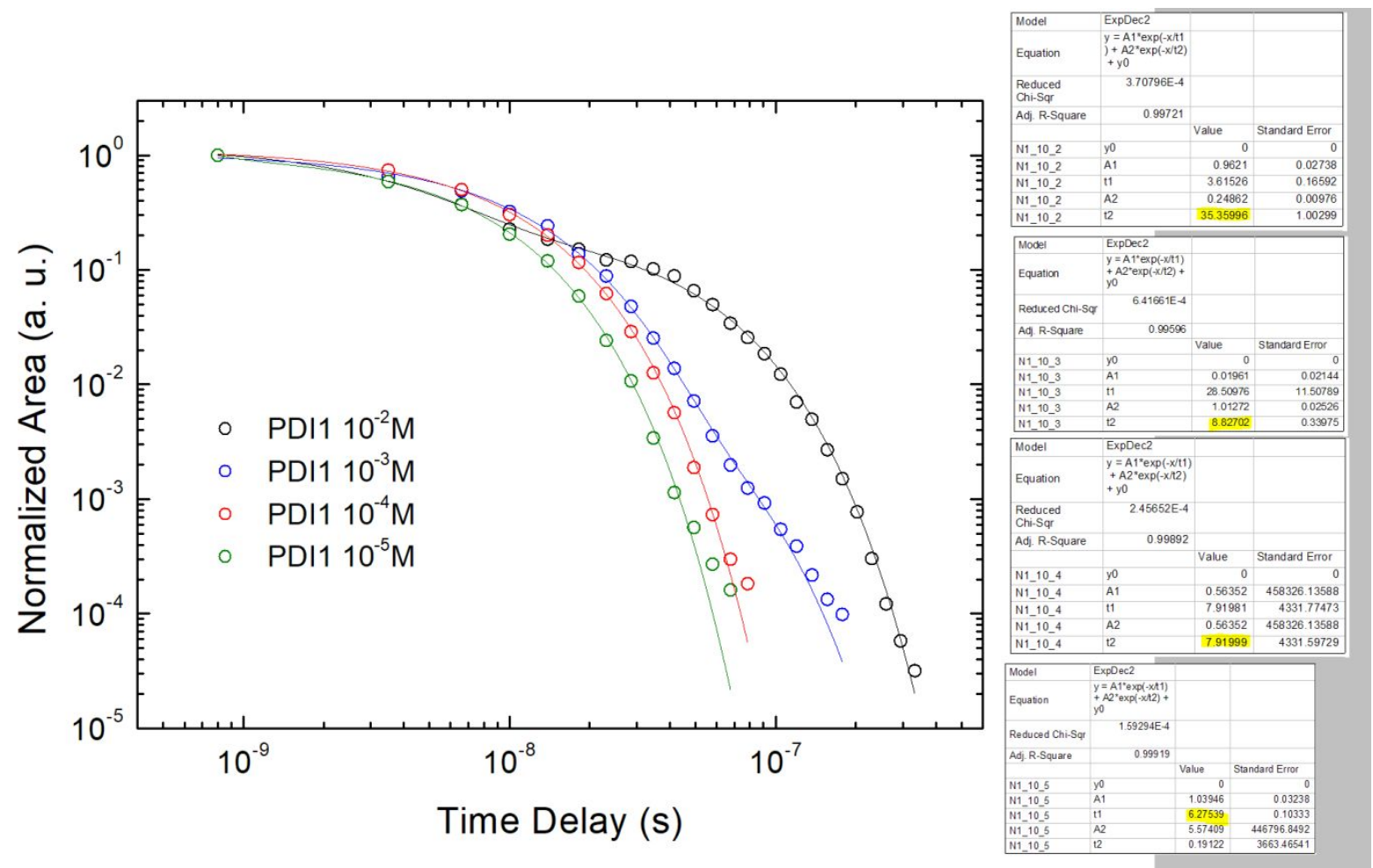

Figure S20: Time-resolved emission decay kinetics of PDI1 solutions.
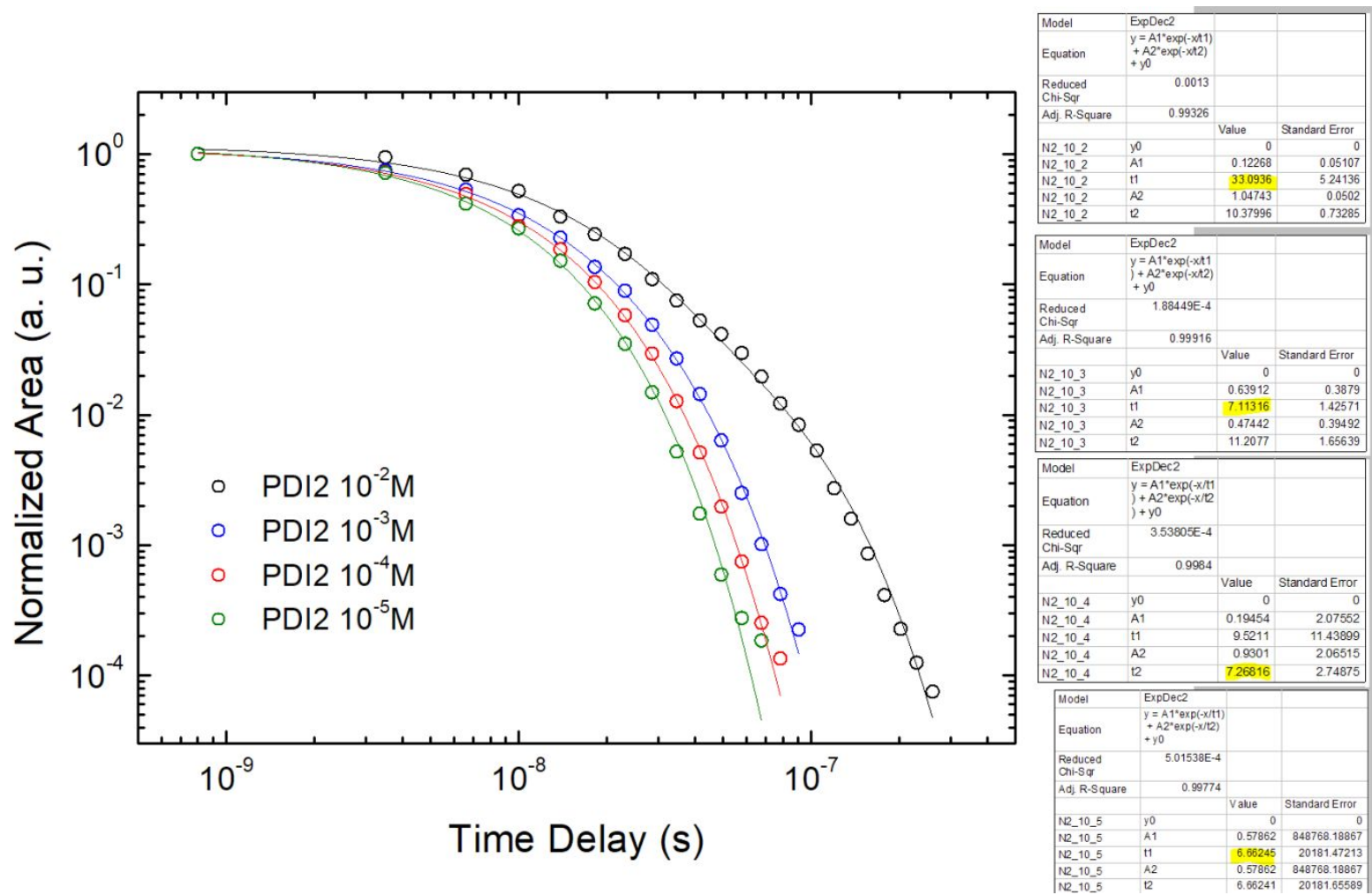

Figure S21: Time-resolved emission decay kinetics of PDI2 solutions. 


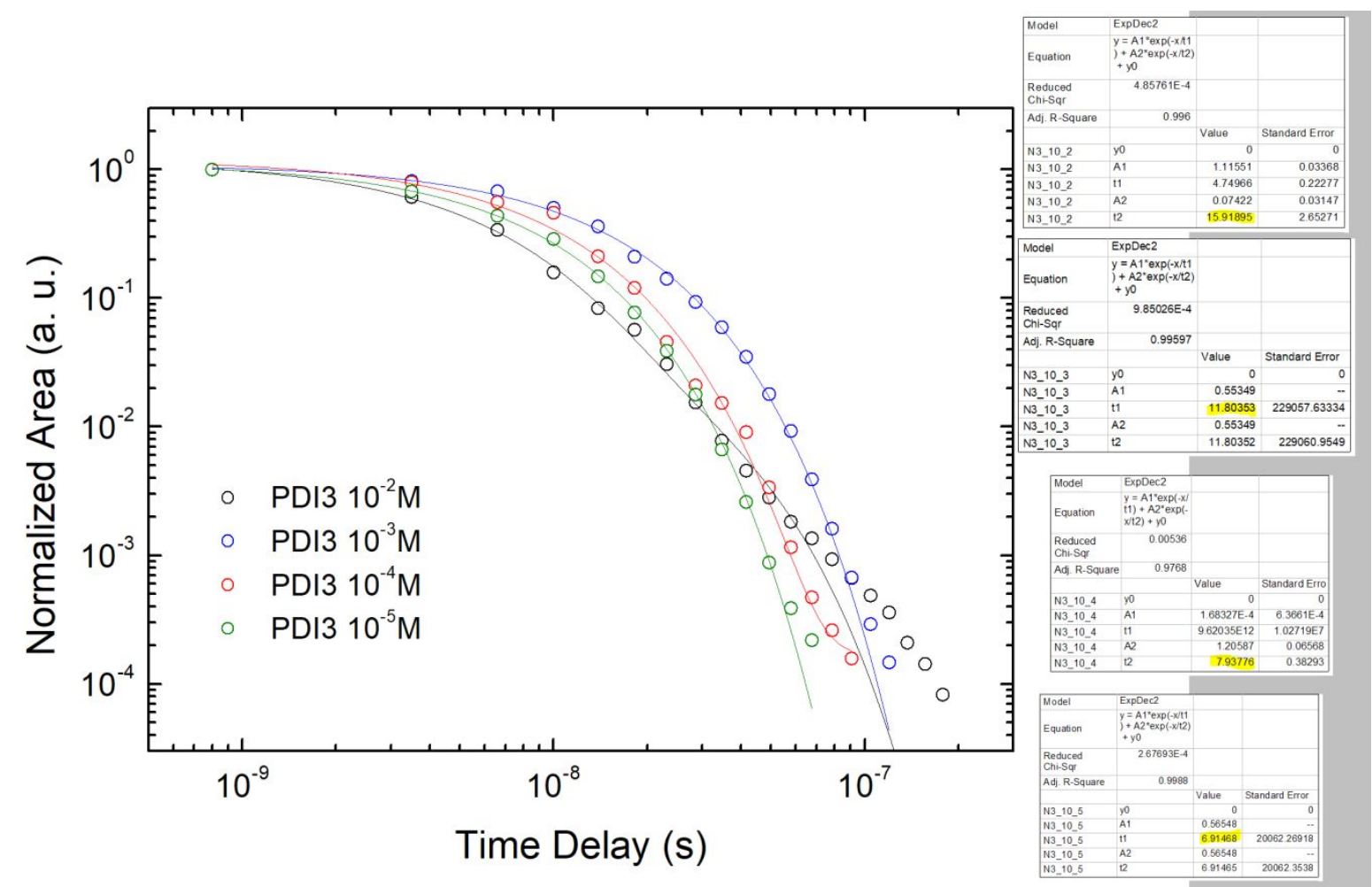

Figure S22: Time-resolved emission decay kinetics of PDI3 solutions.

Table S2: Photoluminescence quantum yields of PDIs neat films (spincoated) ( $\lambda_{\text {ext }}: \mathrm{S}_{0^{-1}}$ electronic band, i.e., $490 \mathrm{~nm}$ for PDIref and $512 \mathrm{~nm}$ for PDIs1-3).

\begin{tabular}{|c|c|c|c|c|}
\hline & PDIref & PDI1 & PDI2 & PDI3 \\
\hline PLQY (\%) & 2.1 & 2.4 & 16.8 & 15.0 \\
\hline
\end{tabular}

Table S3: Average lifetime (ns, weighted average of biexponential fit) for PIA peaks of PDIs at $\left[10^{-4}\right]$ and $\left[10^{-3}\right]$ concentrations.

\begin{tabular}{|c|c|c|}
\hline & \multicolumn{2}{|c|}{ Concentrations } \\
\hline & {$\left[10^{-4}\right]$} & {$\left[10^{-3}\right]$} \\
\hline PDI1 & $4.4 \pm 0.3$ & $7.4 \pm 0.1$ \\
\hline PDI2 & $5.2 \pm 0.04$ & $7.58 \pm 0.04$ \\
& & $8.5 \pm 0.1$ \\
\hline PDI3 & $12.2 \pm 2.7^{*}$ & extincton
\end{tabular}

* outlier with larger error possibly due to weaker sample optical density (see extinction coefficients Figure S10) 


\section{References}

(1) Chen, S.; Liu, Y.; Qiu, W.; Sun, X.; Ma, Y.; Zhu, D. Oligothiophene-Functionalized Perylene Bisimide System: Synthesis, Characterization, and Electrochemical Polymerization Properties. Chem. Mater. 2005, 17 (8), 2208-2215. https://doi.org/10.1021/cm048642z.

(2) Sriramulu, D.; Valiyaveettil, S. Perylene Derivatives as a Fluorescent Probe for Sensing of Amines in Solution. Dye. Pigment. 2016, 134, 306-314.

https://doi.org/10.1016/j.dyepig.2016.07.028.

(3) Huang, Y.; Zhang, W.; Zhai, H.; Li, C. Alkylsilane-Functionalized Perylenediimide Derivatives with Differential Gas Sensing Properties. J. Mater. Chem. C 2015, 3 (2), 466-472. https://doi.org/10.1039/c4tc02059f.

(4) Jiménez, Á. J.; Spănig, F.; Rodríguez-Morgade, M. S.; Ohkubo, K.; Fukuzuml, S.; Guldi, D. M.; Torres, T. A Tightly Coupled Bis(zinc(11) Phthalocyanine)-Perylenediimide Ensemble to Yield Long-Lived Radical Ion Pair States. Org. Lett. 2007, 9 (13), 2481-2484. https://doi.org/10.1021/o10707968.

(5) Zeng, C.; Meng, D.; Jiang, W.; Wang, Z. Synthesis of Isomeric Perylenodithiophene Diimides. Org. Lett. 2018, 20 (20), 6606-6609. https://doi.org/10.1021/acs.orglett.8b02983.

(6) Aksoy, E.; Danos, A.; Varlikli, C.; Monkman, A. P. Navigating CIE Space for Efficient TADF Downconversion WOLEDs. Dye. Pigment. 2020, 183 (May), 108707. https://doi.org/10.1016/j.dyepig.2020.108707.

(7) Guner, T.; Aksoy, E.; Demir, M. M.; Varlikli, C. Perylene-Embedded Electrospun PS Fibers for White Light Generation. Dye. Pigment. 2019, 160 (August 2018), 501-508.

https://doi.org/10.1016/j.dyepig.2018.08.040.

(8) Yan, Q.; Zhao, D. Conjugated Dimeric and Trimeric Perylenediimide Oligomers. Org. Lett. 2009, 11 (15), 3426-3429. https://doi.org/10.1021/o19012734. 